\title{
Implementing Conversational AI in Libraries: A practical approach
}

\author{
Subhajit Panda \\ Assistant Librarian, \\ University Library, Chandigarh University, \\ Mohali, Punjab \\ Email: subhajit.e9641@cumail.in \\ Rupak Chakravarty \\ Professor, \\ Department of Library and Information Science, \\ Panjab University, Chandigarh \\ Email: rupak@pu.ac.in
}

\begin{abstract}
:
The outbreak of present pandemic Coronavirus (official designation COVID-19 or 2019-nCov) forced us to adopt "new normal" and give a greater use and reliance on virtual space than ever before with physical spaces. In the context of the library, shifting of the user assistant programme from physical to virtual face a problem in this transition. This paper based on the probable solution with the use of practical implementation of conversational AI i.e. "Bot" or "Chatbot" to fulfil user needs $24^{*} 7$ without human intervention. A chatbot is a computer program that can simulate conversation and interact with humans spoken, written or both. It acquired a set of pre-programmed commands and continue learning based on the inputs it receives. This paper gives a general overview of conversational AI, Chatbot and its multitasking features and a practical overview of the implementation of one of the famous chatbot provider Kore.ai using its free to use plan. Additionally, the paper explained the requirement of the present situation \& long term benefits of chatbot in the library.

Keywords: Artificial Intelligence, Bot, Chatbot, Conversational AI, Kore.ai
\end{abstract}




\section{Introduction:}

Covid-19 has forced upon us the "new normal" with noticeable changing in the information seeking behaviour (ISB) of the information seekers during this pandemic. Social Distancing is one of the most crucial aspect of thisnew normal. For the librariesworld over this translates into greater use and reliance on virtual space than ever before with physical spaces literally crying for the revival of the old and gold hustle and bustle and user foot-falls. Libraries will have to adopt business process re-engineering and explore and identify innovative solutions for user outreach in future. In this context, it is expected that they will have to develop some kind of knowledge-base (kb) with userinterface bridging the geographical divide. Users' assistance and promotion of library resources are to be taken care of through technological intervention so that users visualize libraries as their allweather companions in meeting their academic pursuits in these difficult times.

\section{Defining a Chatbot:}

A computer program that simulates human intervention to perform a real-time conversation with people and developers (e.g. library users \& virtual reference librarians) in terms of spoken, written or both, with the realization of talking to a human being (Smartsheet Inc., 2020). Chatbots are computer software programs designed to simulate human conversation via text or audio - are emerging as the key mechanism for companies to interact with consumers at scale across these platforms. It is artificial intelligence (AI) software that can simulate a natural language conversation with a user through messaging applications, websites, mobile apps or a telephone (Cordny, 2020).

Michael Mauldin first coined the term "chatterbot" in 1994 and created the first chatbot "Julia" with Verbot, a popular software program and development kit. AI chatbots are also known by many other names today, like; talkbot, bot, IM bot, intelligent chatbot, conversation bot, AI conversation bot, talking bot, interactive agent, artificial conversation entity, or virtual talk chatbot (Smartsheet Inc., 2020).Chatbots are software applications that use artificial intelligence \& natural language processing to understand what a human wants, and guides them to their desired outcome with as little work for the end 
user as possible. Like a virtual assistant for the library user experience touchpoints.A well designed \& built chatbot will:

a) Use existing conversation data (if available) to understand the type of questions people ask.

b) Analyze correct answers to those questions through a 'training' period.

c) Use machine learning \& NLP to learn context, and continually get better at answering those questions in the future.

\section{Conversational AI and Smart Chatbots:}

It refers to the use of messaging apps, voice-based assistants and chatbots to automate communication for personalized user experiences. Conversational interactions support engaging, two-way interactions combined with automation and artificial intelligence (AI) connecting humans and machines through virtual assistants and chatbots. The real power of conversational AI lies in its ability to simultaneously carry out highly personalized interactions with large numbers of individual library users. Conversational AI can fundamentally transform an organization, providing more ways of communicating with library users, while facilitating stronger interactions and greater engagement. An intelligent chatbot possesses learning capabilities using its experience thus improve its performance. Machine Learning is the process of training computers to perform tasks with a combination of data analysis and probability rather than following a strict rules-based approach. Natural Language Processing (NLP)includes two subset fields - natural language understanding and natural language generation (Khurana, Koli, Khatter, \& Singh, 2017).

Warschauer and Healy (1998) define NLP as "the process of a computer extracting meaningful information from natural language input and/or producing natural language output." Deep Learning (also referred to as neural networks) is a branch of machine learning that emulates the interconnection of neurons in the brain. While chatbots are machines that converse with humans via audio or text, AI chatbots are smarter versions of these chatbots. 


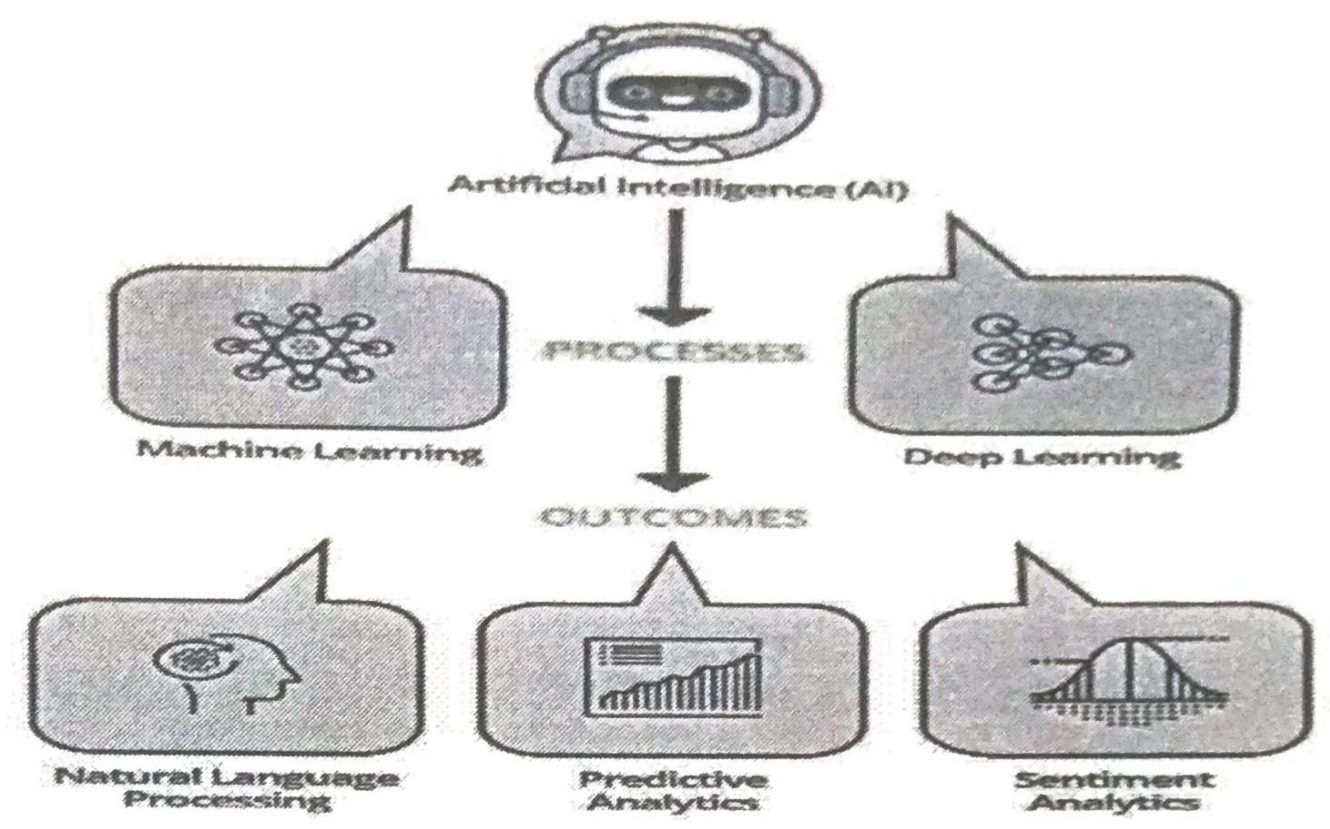

Fig 1: How an AI Chatbot Works

(Source:https://medium.com/marketing-and-entrepreneurship/how-dochatbots-work-459353cb8895)

They use machine learning and natural language processing to understand human intent better. They provide more natural communication with humans and are trainable and, over time, learn and improve communication with your target audience.For every interaction they have with the audience, they become smarter. The bots also become better and more efficient at task completion.AI chatbots are available $24 / 7$ and can handle conversations with multiple library users at once.An intelligent/smart chatbot:

understands user needs and is prepared to meet users' requests.

$>$ Handle different styles and topics of conversation with ease.

$>$ Generate new responses apart from just retrieval from predefined responses.

$>$ Take part in complex, longer conversations.

$>$ Deal with multiple users queries. 

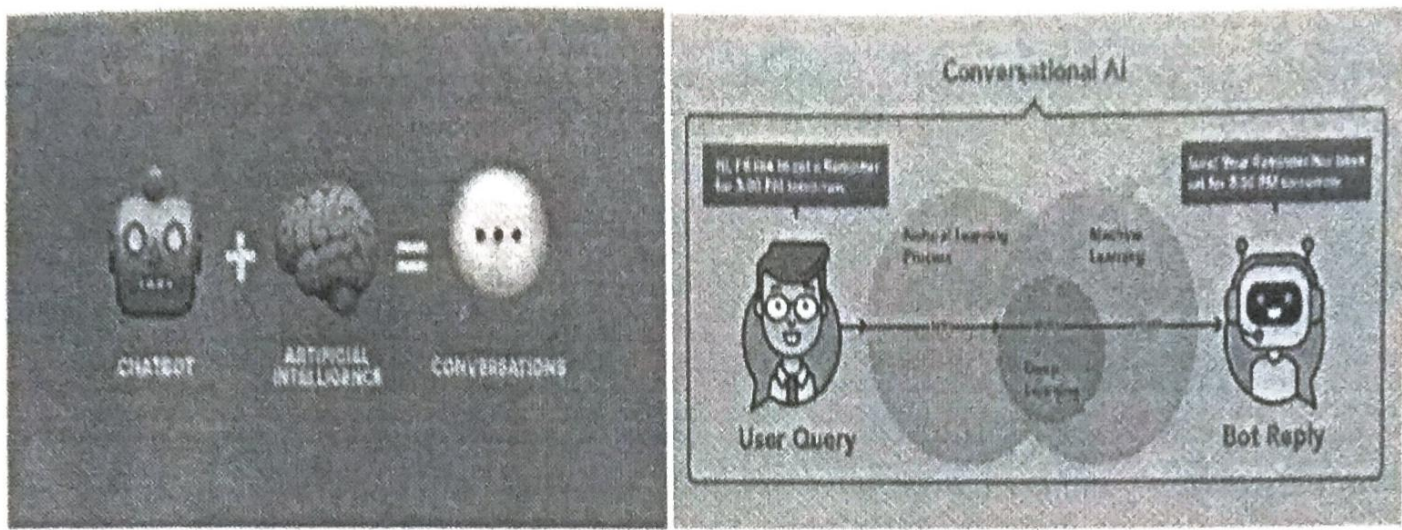

Fig. 2: Conversational AI Architecture: User Query \& Bot Reply

(Source 1:https://www.drift.com/learn/chatbot/ai-chatbots/)

(Source 2:https://www.iotforall.com/what-is-conversational-ai/)

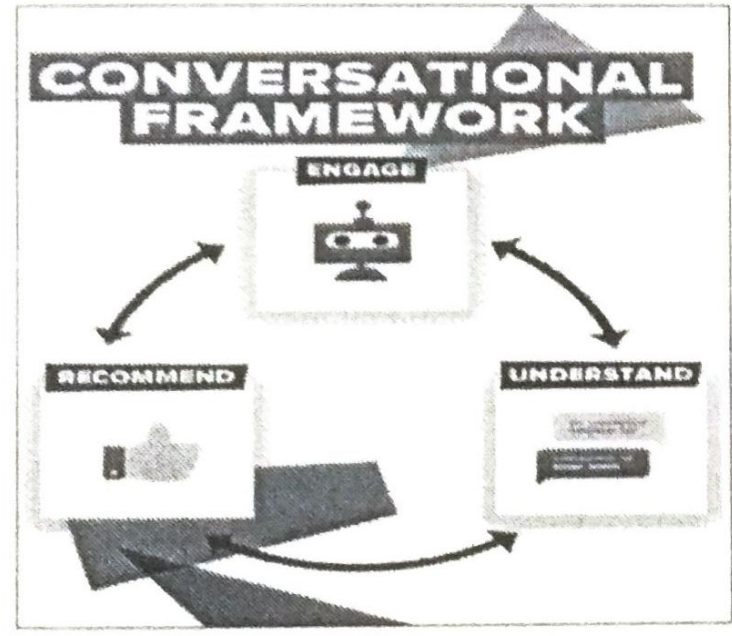

Fig. 3: Conversational

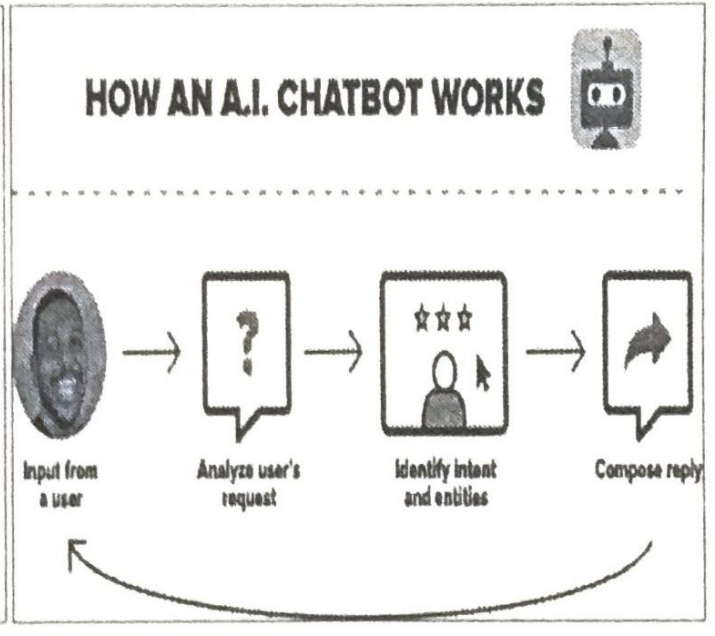

Fig. 4: Working of AI Chatbot

Framework: Engage, Recommend $\&$ Understand

(Source 1: https://blog.quagrowth.com/what-is-conversationalmarketing)

(Source 2:https://www.drift.com/learn/chatbot/ai-chatbots/)

Chatbots streamline interactions between people and services, enhancing user experienceimproving the library users engagement process and operational efficiency by reducing the typical cost of user specific service. To be successful, a chatbot solution should be able to effectively perform both of these tasks. Human support plays a key role here: Regardless of the kind of approach and the platform, human 
intervention is crucial in configuring, training and optimizing the chatbot system.

Artificial intelligence (AI) makes it possible for chatbots to "learn" by discovering patterns in data. Then these chatbots will apply the pattern to similar problems or slightly different questions without instruction. Artificial Intelligence enables the skills to perform tasks, solve problems, manage information and process knowledge without human intervention (Smartsheet Inc, 2020). AI has made chatbots more lifelike than ever before, and they are becoming pervasive. To maximize the ability of artificial intelligence (AI) chatbots, to improve its services, to make it more cost-effective, and to increase users engagement, organizations need to understand their basic objectives before implementing a chatbot and make invest on it.

\section{Features of Free AI Chatbot:}

\section{A. Provide a virtual welcome mat to the users:}

Chatbots are the perfect way to welcome new users to the website, by providing a friendly greeting on their arrival, guiding them through the site and helping them find the information that they need. There are three common way to welcome,

(i) Provide a concise welcoming statement

(ii) Let them know what they can do

(iii) Tell them how to get help

\section{B. Bring library users and resources together through personalized, omnichannel experiences}

Build a virtual assistant, enabling library users to find resources with few clicks, and instantly access them in the library websites. Through virtual assistants, users can interact with their query, navigate through websites, access documents, and continue the same conversation across supported channels in multiple times as they required (Kore.ai, 2020a). Some notable omnichannel approaches are,

a) Virtual presence: be everywhere with the library users are and deliver the experiences they expect. 
b) Cross-Channel Conversations: switch back and forth between channels without losing the context of the conversation.

c) Cross-Channel API Handling: connect virtual assistants to select channels through automatic handling of connectivity requirements via APIs, e.g. linking to Facebook, Email, SMS etc.

d) Automatic Message Formatting: the connectors automatically adapt messages to match the unique formatting used by each communication channel.

e) Minimalistic Coding: with pre-built channel connectors, developers can quickly and easily deploy VAs across channels.

f) Location Services: enhance channels in GPS-enabled devices by building chatbots to perform tasks based on a user's location.

g) Agent Handoff: chatbot platform provides pre-built integration with live agent software, so conversations can be seamlessly transferred from virtual assistant to human agent. (Kore.ai, 2020a)

\section{Engage library users across every language, culture, and locale:}

Chatbot allows librarian to create multilingual virtual assistants that speak to users in their native tongue, eliminating language barriers and making the localization process easier, more effective, and more affordable. Multilingual features of chatbot includes,

a) Build Multilingual Virtual Assistants: virtual assistants of chatbot auto-detect and switch language based on user utterances.

b) Custom-Named Entity Recognition: virtual assistants can be trained to recognize and automatically extract custom entities in any configured language.

c) Automatic System Entity Recognition: virtual assistants recognize universal library standards like title, author, subject, classification number, dates of publication, date of entry, and more, without the need for additional training.

d) Built-In and Custom Small Talk: virtual assistant platform automatically responds to user small talk, including welcome 
messages and other forms of chit-chat, for all enabled bot languages.

\section{Universal Bots: Orchestrate Tasks From Multiple Specialized Virtual Assistants}

Provide library users with a consistent IVA experience by linking individual (standard) virtual assistants together to create a Universal VA. Allow library users to access multiple virtual assistants from a single chat and bot persona. This Universal Bot,

a) acts as a single, unified interface for virtual assistant users.

b) allows VAs to be built independently for specific use cases and then linked together to form a Universal bot.

c) performs intent recognition across all linked VAs to understand the user's intent, ranks them based on relevance, and engages the appropriate VA to perform the task.

d) handles ambiguous, unrecognized requests by providing the user with matching bot names, tasks, or suggestions so they can quickly make the intended selection.

e) can be deployed to the same or different communication channel as its linked virtual assistant.

f) allows developers to view and analyze all user interactions with the Universal VA and test them in batches to discover single matched intents, multiple matches with the same score, and unmatched intents. (Kore.ai, 2020e)

\section{E. Knowledge AI: Transform Unstructured and Structured Data Into Actionable, Identifiable Q\&As}

Effortlessly move existing FAQ content of the library website into the virtual assistant knowledge base. Knowledge AI automatically extract, review, analyze and publish FAQs from user enquiry and online reference services, with zero coding. Knowledge AI worked in three steps, answer user questions with the help of existing wealth of information available in the library website pages, documents from the cloud drives or institutions content management system. 
- extraction engine allows librarians to quickly and easily extract and synthesize information and use that to train virtual assistant that answer FAQs automatically and intelligently.

- if any new query arises by the users, Knowledge AI automatically identified it, transferred it to the library professional for answer manually, and add it to the knowledge base. Thus the knowledge base of chatbot become richer with the time.

\section{A. Auto Dialogue Generation:}

Chatbot create intelligent virtual assistant from scratch using transcripts. It empower virtual assistants to have complex and meaningful conversations with users at scale to create dialogs that match the flow of natural, human conversations. The Chatbot platform follows a step-by-step approach to accurately extract dialog and train the virtual agents from chat transcripts.

a) Identify relevant intents: It is the first step in the dialog generation process based on normalizing the utterances, extracts relevant utterances from the same conversation transcripts which can be complex with primary intents, supplemental intents and discover special features with unsupervised machine learning algorithms that are used to cluster semantically similar utterances.

b) Discover intent discourse patterns: Identifying discourse patterns for each intent is the second step in the dialog generation process. A conversation typically will have a collection of sub-tasks which can be categorized as inputs/responses required to fulfill the task. Chatbot extracts various sub-tasks and valid ordering of these in an unsupervised manner that captures the variability across conversations.

c) Generate VA training data: Generate training data set to recognize intents, supplemental intents, and also populate bot response messages for entity prompts, confirmations and other messages.

d) Model VA dialog tasks: The final step in the auto dialog generation process involves extracting and assigning entities to each identified intent. A dialog is generated after sequentially arranging all the combined intent and entity groups, and is based on their occurrence. 
The bot model is then trained with the new dialogs before being imported into the chatbot dialog builder.

e) Import into VA/App builder: Bot modeled for conversation flow and along with training utterances can be imported into bot builder. From here, developers can visualize and review conversation flows and branches using the visual dialog editor. This provides a solid foundation from which to build - or expand on - the virtual agent's conversational capabilities.

\section{Practical Implementation:}

The practical part of the paper consisting of explaining detailed technological aspects of one of the most famous chatbot application,

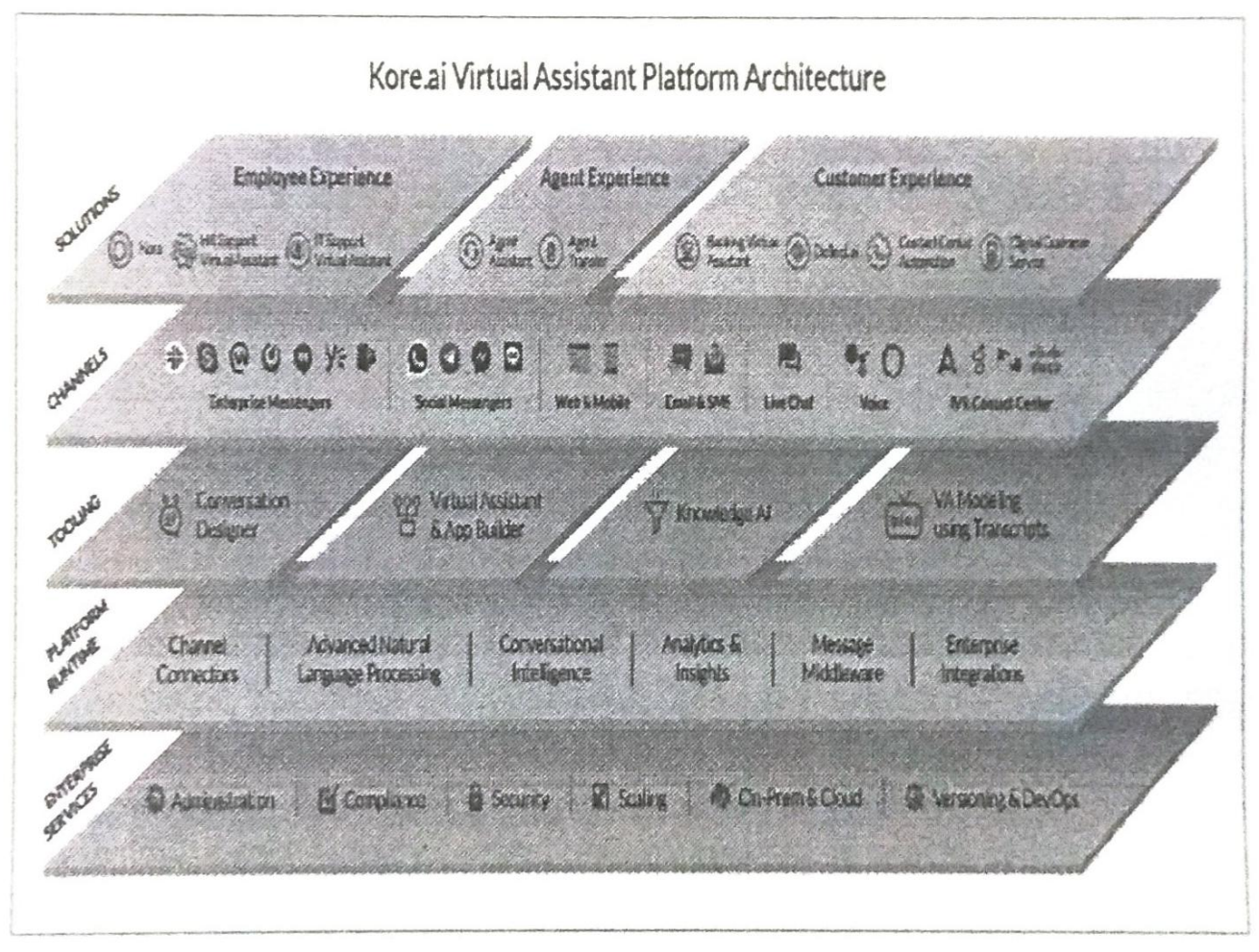

Fig. 5: Kore.ai Virtual Assistant Platform Architecture

(Source:https://kore.ai/)

"Kore.ai", which provides all of the components and capabilities that required to build sophisticated virtual assistants that can satisfy small community of school library to complex network of an university library, accelerate the time for communication, and exceed user expectations. 
In build AI-powered virtual assistants of Kore.ai can meet unique operational requirements and perform multiple roles across diverse industries, e.g. Banking, Insurance, Telecome, Airlines, Retail, Travel \& Hospitality, Healthcare, Media \& Entertainment, Educational Institutions, Public Libraries etc. It is recognized by global analyst firms, trusted by leading companies, adopted by Independent Software Vendors (ISVs) and System Integrators (SIs), and admired by developers throughout the world. Moreover like an efficient smartbot, Kore.ai also possess following functions,

- Multipronged NLP Engine: Enables precise intent identification

- Flexible Deployment: On-premise, cloud or hybrid model

- Conversational Intelligence: Humanizes user-bot conversations

- Dashboard and Analytics: Get the insights needed to refine VA performance and the user experience

- Omnichannel Platform: Supports over 30+ channels so librarian can reach toward every library users wherever they are

- Open and Extensible: Allows integration with custom channels and custom ML models (Kore.ai, 2020c)

To get a new bot in Kore.ai user have to go through some specific stages and choose some options as per the requirements.

\section{(a) Stage 1: Choice of requirements for build a VA:}

In order to create a bot first, we must go through "Create a Virtual Assistant" on the Kore.ai website homepage, which will guide us to the login page. If anyone want to get some knowledge before starting there also option for "Get Trained" and "Request a Demo". After log in or create an account on Kore.ai, the user need to select in which pathway he/she want to create a bot. It depends upon his/her previous knowledge and ability of bout-building,

I'm new to bots, but eager to learn: Learn all about bots and key development concepts. Begin the journey by building a very first conversational bot. 
- I'm familiar with bots, and I'm ready to start building: Take a step-by-step, guided tour of Kore.ai platform and learn how easy it is to build bots with Kore.ai.

- I'm a bot expert, and I'm ready to roll: Jump straight into the platform and start creating memorable conversational experiences.

For beginner it's better to choose the first or second option because it gives a five step guide about how to create a new bot and virtual assistant using Kore.ai.

> Step 1: A quick introduction to the world of bots

> Step 2: A brief overview of key bot concepts

> Step 3: A short review of the bot building process and best practices

> Step 4: A look at the Kore.ai bots platform

> Step 5: Let's get started by building your first bot

\section{Stage 2: Choice of creation of a new Bot:}

For create a new bot first we have to choose between "Create your first bot", "Install smart bot", Install sample bot" and "Import a bot". Fore create a new bot the "Bot Name", Default Boat Language" and "Boat Type" have to be selected. For first time user "Standard Bot" is the best option, but there also two other option i.e. "Universal Bot" and "Smart Bot" if required.

\section{(b) Stage 3: Bot Tasks:}

A Kore.ai bots can perform a wide variety of simple to complex tasks by having interactive conversations with library users. To build the virtual assistant more powerful, after the creation of a bot, we need to implement different type of bot tasks either by manually enter bot tasks or extract from URL or imported file from desktop. Following are the tasks which need to be selected and performed as per the requirement of the library, 
> Dialogue Tasks: A dialog task is a graphical representation of the conversation between library user and the Bot, which is a network of nodes connected using conditions.

- Knowledge Graph: Build the bot's knowledge Graph by adding FAQs or by importing from file.

Alert Tasks: The user can setup alert tasks to get notified when events occur in the source system. Once setup, these tasks poll an API (or wait on a webhook) to fetch events and notify the library user with an alert.

> Small Talk: Small Talk is a casual conversation that the library users can have with the bot. The conversation can be designed a series of volleys between bot and the user (Yerram, n.d.).

> UI Forms: Transform lengthy, routine conversations into engaging activity using UI Forms. Design forms to capture inputs from library users and add them to Dialog Tasks or Panels.

> Digital Views: Design and launch interactive views to provide a rich visual experience to library users. Widgets can proactively present relevant information to library users and also allow them to swiftly take any follow-up actions by directly conversing with your bots.

- Information Tasks: Information tasks are simply presents structured information in the form of report to the library user as a link. "General Enquiry", "Library Guide",

"Book Return Policy" etc are examples of report tasks.

>Action Tasks: Actions are simply, anything the bot can "do" when specifically asked to do it by the library user. Actions are API driven and act as a powerful connection to libraries backend systems.

Flows: Alerts produced by Alert Tasks can be connected and mapped to Action Tasks allowing for mini workflows and even bot to bot data transfers. 
(c) Stage 4: Import FAQs and Prepare Knowledge Graph

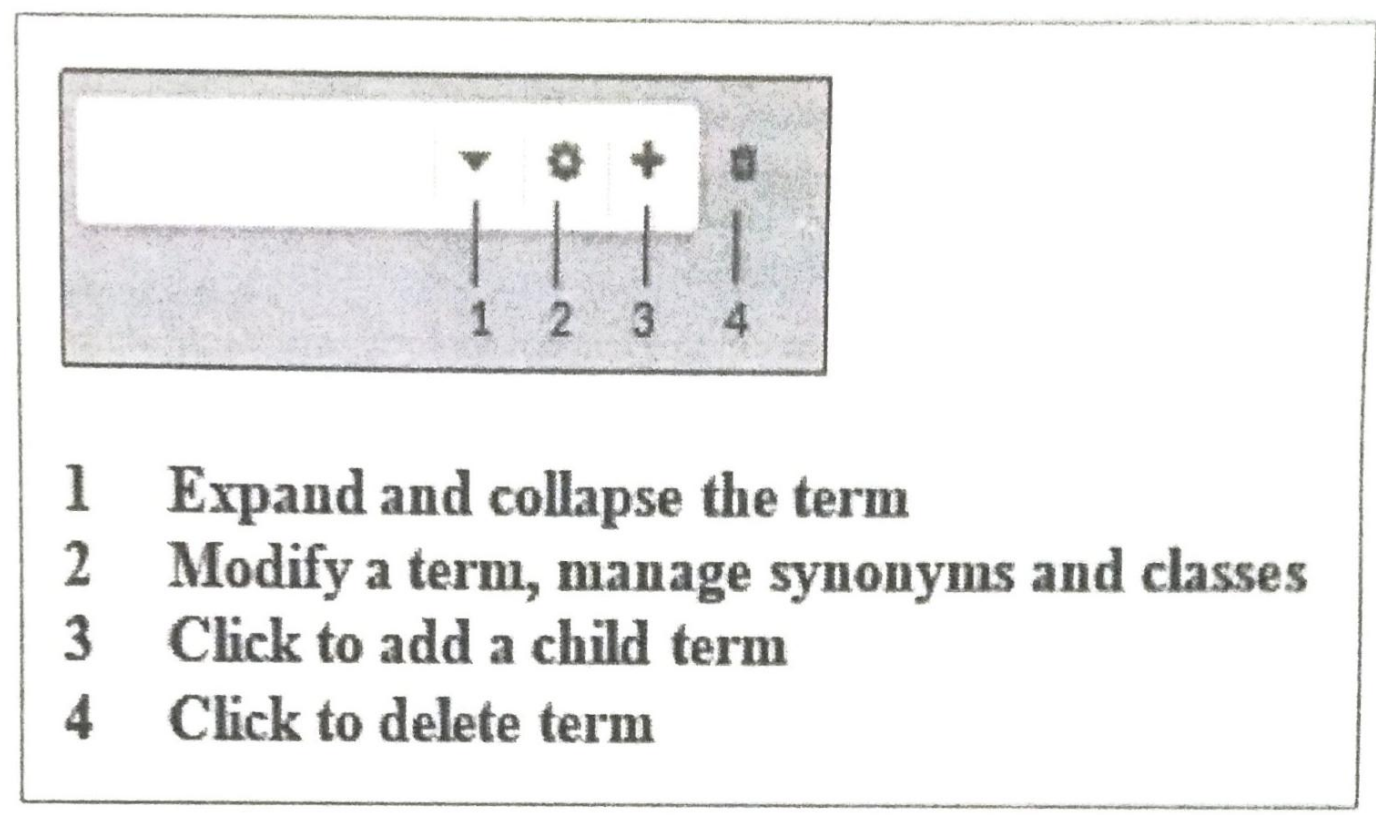

The knowledge graph is the process of enabling semantic intent detection by defining bot ontology structure and connecting either the Dialog Tasks or by defining questions and answers. Among the abovementioned tasks, the building of the bot's knowledge graph by adding FAQs either by "Add Intent Manually" or by "Add from extraction" is the most important and of maximum value to rich the knowledge base of the bot. This extraction can be done either by "Extract from file" via drag and drop or browse any PDF or CSV file from the system and "Extract from URL" method works through the extraction of FAQs from the previous library website or parent institution website or other FAQs channel through Web URL.

Knowledge Graph of chatbot contains some basic features to manage FAQs after they are added as an intent. It is important to note that both the extraction process (i.e. either manually or through URL) goes through a review process before they are added to the FAQs of the Knowledge Graph.

\section{(d) Stage 5: Test the Bot}

After adding intent as FAQs in the Knowledge Graph it is important to check whether the Bot is performing well with those FAQs or not. The testing procedure was done in two ways. First, either via "Utterance Testing" or via "Batch Testing" to test \& train the bot to understand different user utterances and match them with intents and entities. 
Training allows librarian to look into the mind of the bot and fine tune how it understands the library users and train it if necessary. Second, directly put one of the added FAQs in chat session and check whether the bot replies the correct answer or not.

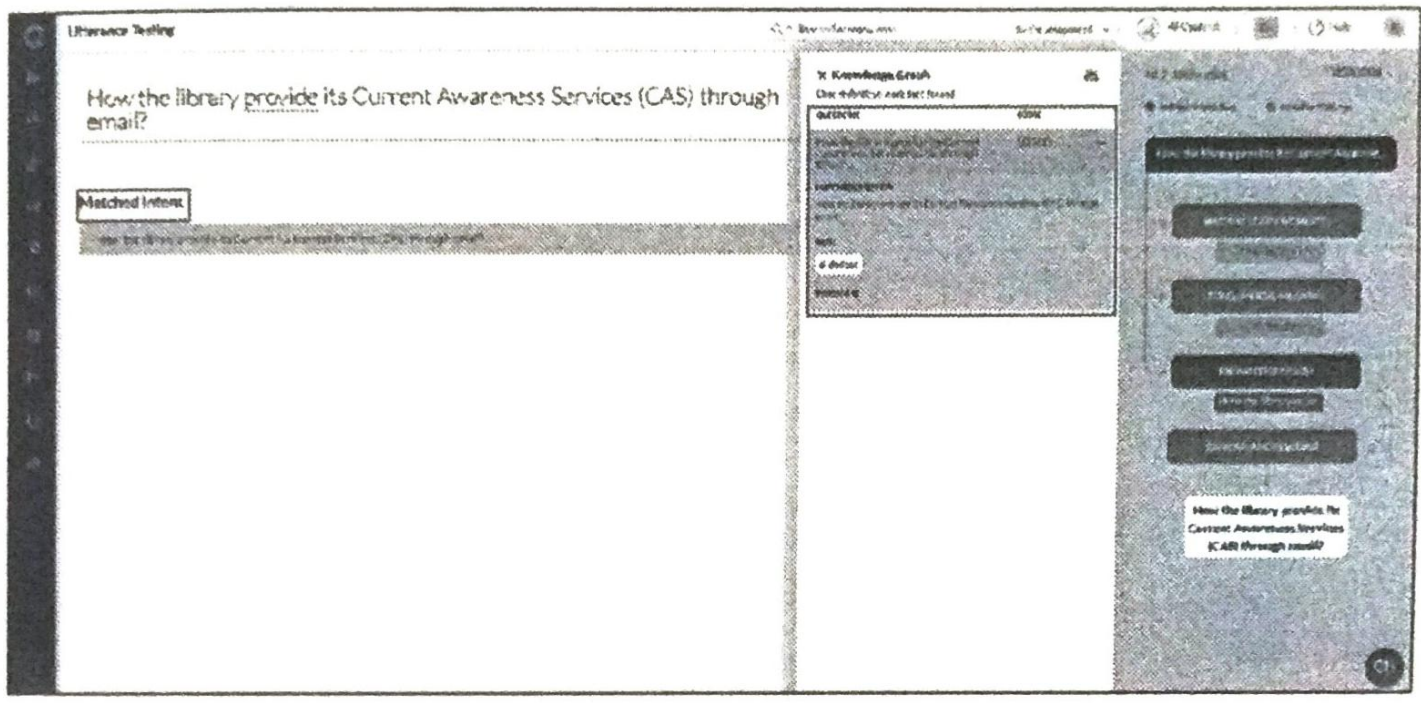

Fig. 6: Utterance Test Result (Bot Replied Successfully)

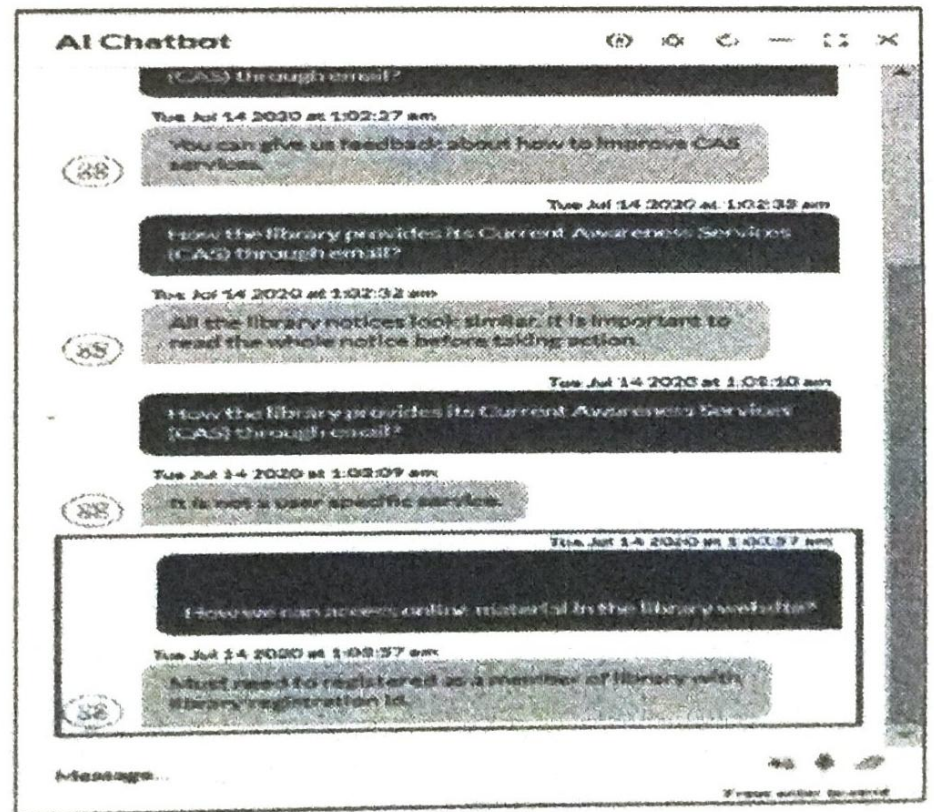

Fig. 7: Check Bot Directly Through a Chat Session (Bot Replied Successfully) 


\section{(e) Stage 6. Small Talk: Greetings}

Small Talks are some casual conversation between the library users and the bot. The conversation can be designed a series of volleys between the bot and the user (Yerram, n.d.). In Kore.ai bot, there are almost 10 inbuilt small talks and also the option to edit them and add a new one. The figure displays a web interface of that small talks where "User Says" denotes the user end conversation and "Bot Says" implies bot's replies.

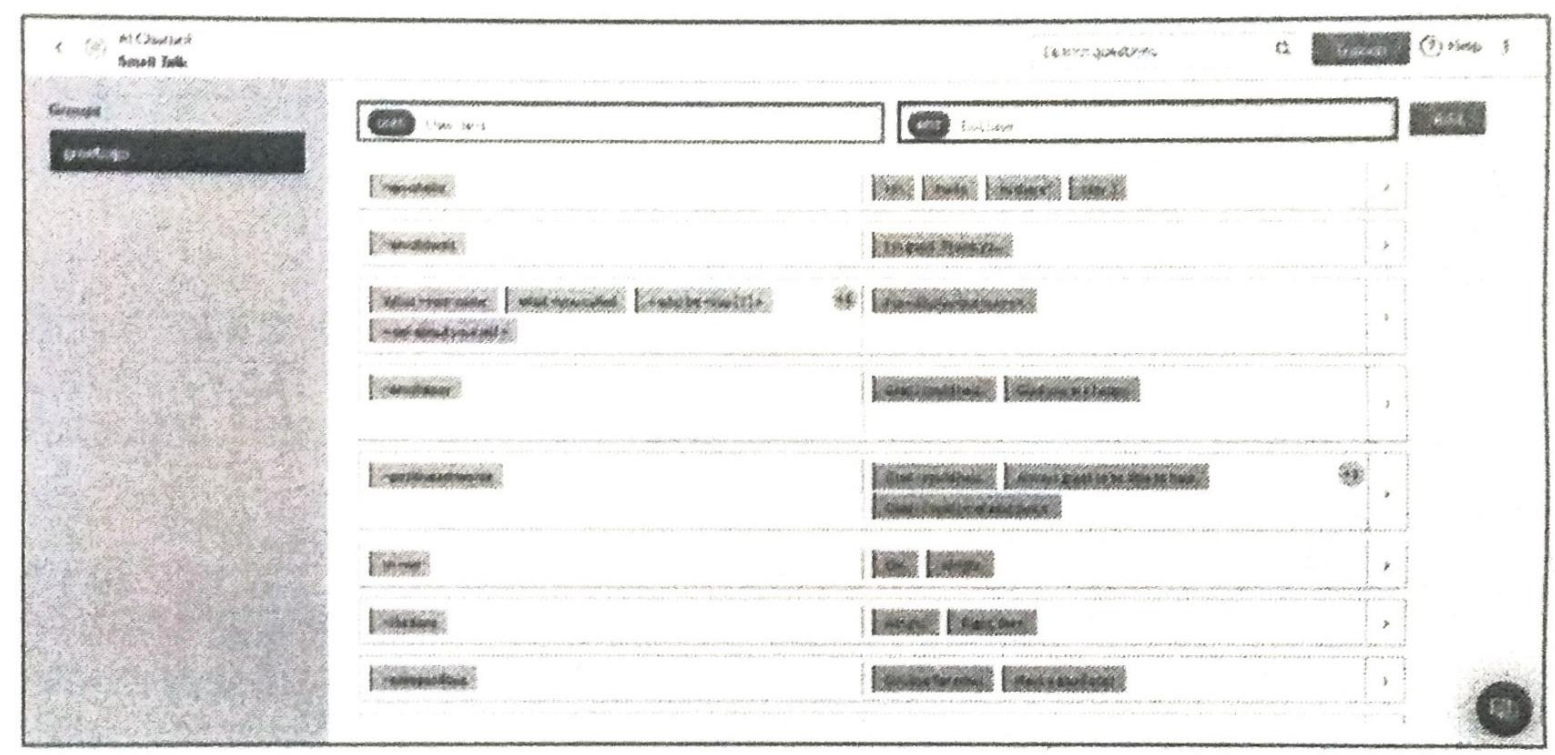

Fig. 8: Small Talk Interface of Bot

\section{(f) Stage 7: Enabling a Channel}

After defining a custom bot, it is now possible to add the channel to the bot to enable end-user accessibility and interaction with the bot when it is deployed. By default, a bot and any defined notification or action tasks are not available to users in any channel unless it is customized for the bot. To add channels it is required to go through the Channels tab of the bot and select an intended channel and enable it upon their requirement based upon their functionality and usage. After enabling a channel "Bot Admin Approval"is required to approve the channel as a part of bot publishing.

To enable one or more channels for the bot, follow these three steps: 
1. Open the bot for which admin want to add the channels,

2. Hover the move of the crusher over the side navigation panel, and then click Channels tab.

3. Click the name of the channel to add it on the page. It opens the instructions page to install the channel.

After the completion of the channel configuration, now it is necessary to connect Kore.ai bot with that particular channel to test it. (Kore.ai, 2020b)

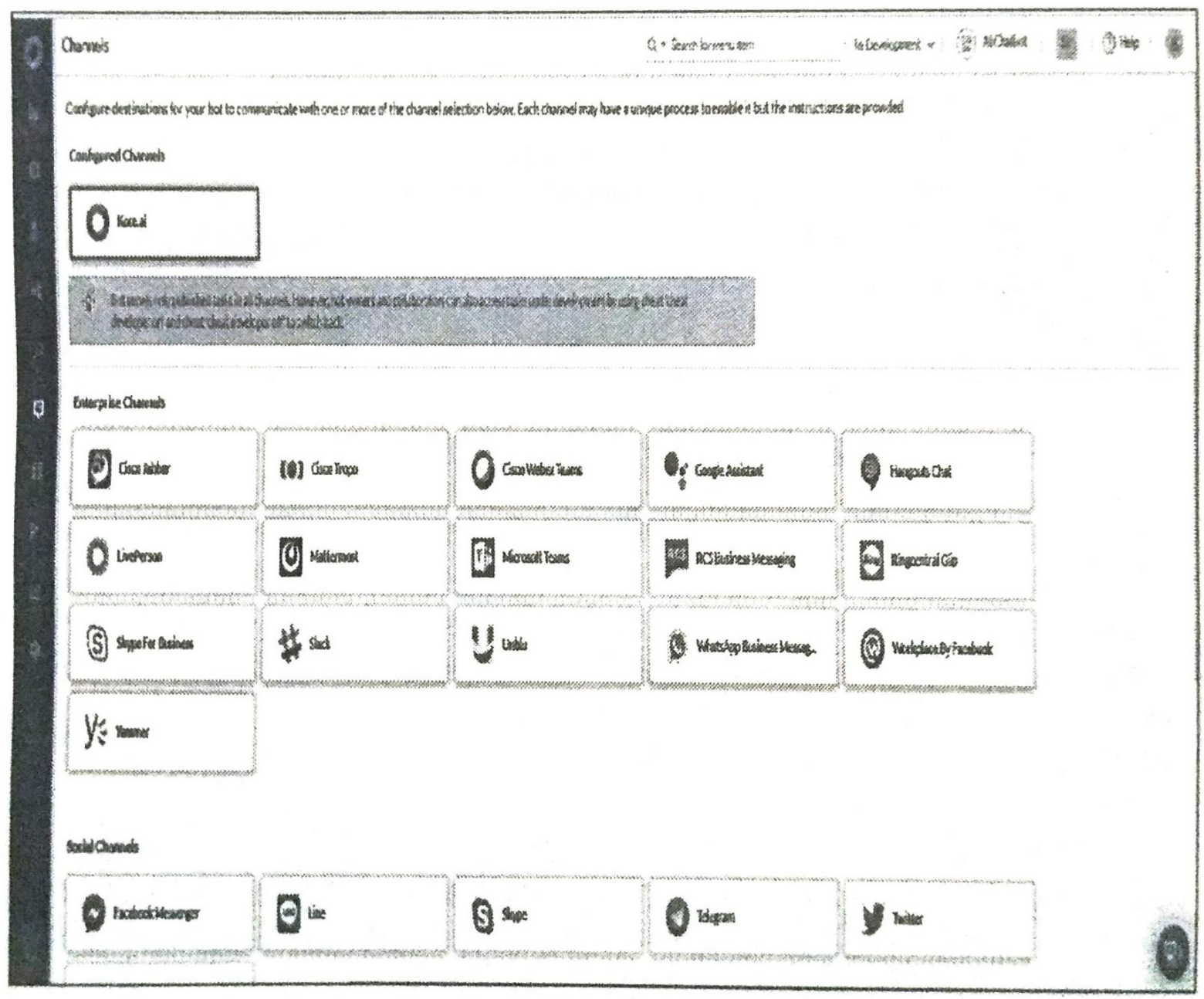

Fig, 9: Configured Channel Kore.ai for Bot Publishing

\section{(g) Stage 8: Publishing the Bot}

End users can interact with a bot only when it is published. When a library professional publishes a Bot task, the platform initiates a request 
to the Bots Admin (Librarian) to approve it. In "Publish" tab of the bot there are some publishable components e.g. "Tasks", "Languages", "Natural Language", "Channels", "API Extensions" and "Settings"; that impact end-user interactions or experience go through the publishing workflow in the Bots Platform.

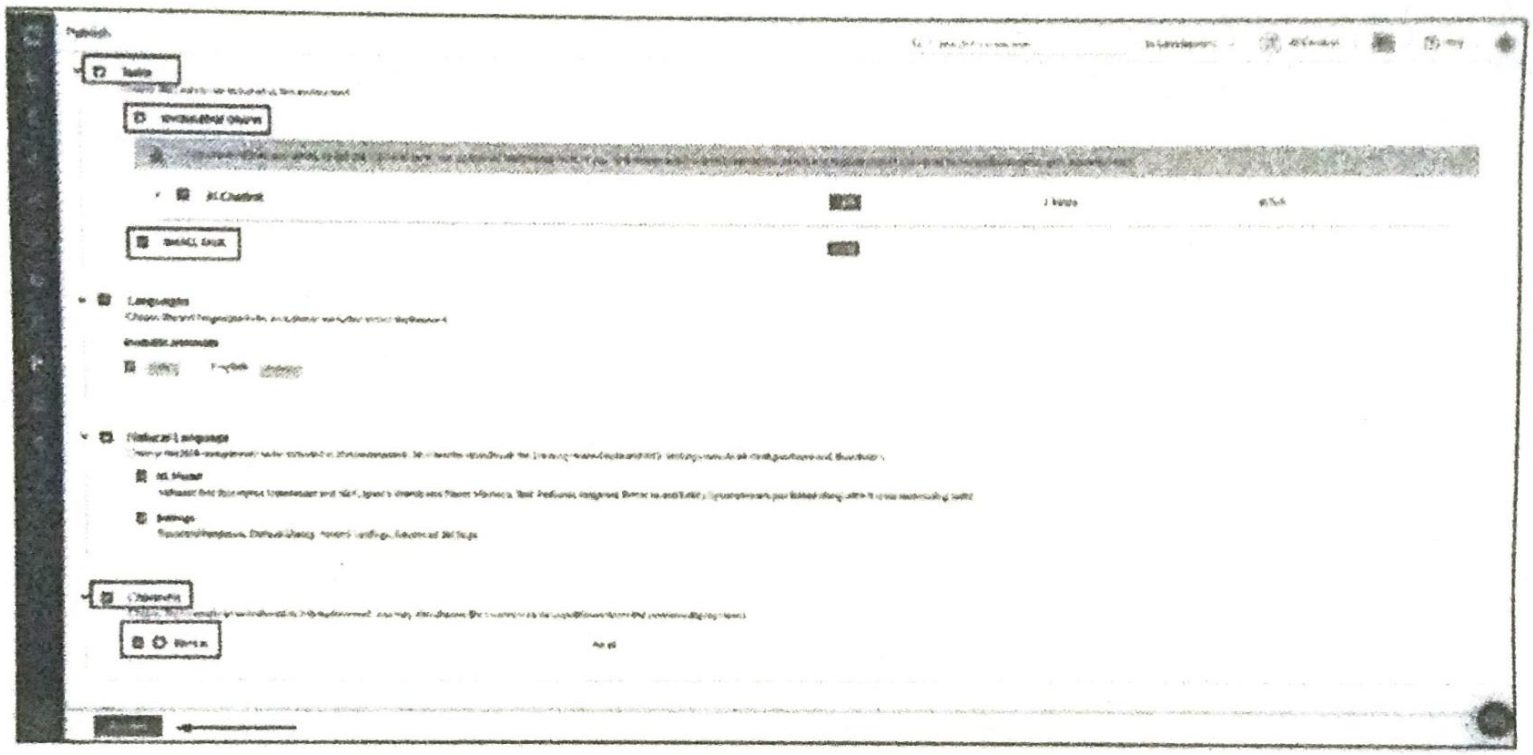

Fig. 10: Bot Publishing Platform

In the present study we create a Bot with the name "AI Chatbot" and configure only two essential tasks, first add intent in knowledge graph as FAQs and secondly configure Kore.ai channel for publishing the bot. In the bot publishing page both the works are selected with small talk as an auto configure tasks in Kore.ai bot for get published. By clicking "Proceed" the bot is publishing under the free tier with 3000 session. The Bots Admin needs to select library users from the institutions Koreai account (default) who can interact with the particular bot, and then approve the publishing request. Once that's done, the selected library users can use the bot in the selected bot channels. (Kore.ai, 2020d)

\section{Chatbots in the Library:}

Chatbots answer basic, always-asked questions. A library chatbot could answer a variety of questions, like:

Questions about hours, locations, and upcoming events. Maybe it could even capture event registrations. 
$>$ With a good API connection, a chatbot could tell a library user when their books are due, or take fine payment information.

Facebook Messenger chatbots. You could build your chatbots to operate within Facebook Messenger, If you get a lot of questions there, it's definitely something to think about.

$>$ Chat-based reference. Have a chat-based reference service? It's very possible your chatbot could answer basic questions, freeing up your staff's time to answer harder questions or focus on other tasks. (King, 2018)

Technology can play a vital role in helping the libraries in meeting any shortage of reference staff as well as shortage of funds. AI chatbots can facilitate online patron account management, self-registration, and selfserve checkout stations (McNeal \& Newyear, 2013). They can provide basic library information like opening hours, etc.on the fly. They can guide the patrons regardingspecific information the are looking in a self-service mode without requiring human intervention through their user-friendly interface embedded on the library website.

The AI powered smart chatbots can also perform searches in the databases based upon the user information demand expressed via Q-A dialogue session. Conversation logs can be analysed to monitor the user ISB and to update the KB (responses). Thus they offer a convenient and helpful way to the patrons who unaware or less aware with the library and its services. Training the library chatbot with the links to the e-resources including OPAC, online databases, and other library information would be of great help and a game-changer in the current pandemic and emerging scenario of post-Covid-19. This will also lead to enhanced use of library resources. Patrons can enjoy personalized user-experience (UX) starting with welcoming them through meaningful greetings.

Al chatbots deployed in the libraries (academic or public) can ease-out any confusion which a general user might face while accessing the library website owing to their complex structure comprising of huge variry of information they contain. Conversational Smartbots can facilitate easy navigation simplifying their information seoking task to a great extent. The responsive interface of the chatbots responds to the 
patron's initial queries while leading to the user satisfaction by adopting a basic-to-advanced in-depth approach/algorithm.

Chatbots can also ease the burden of basic/routine queries so that library staff can focus their attention on more sophisticated queries and providing innovative and value-added services (McNeal \& Newyear, 2013). They can act as a backup information services during unstaffed environments. They make sure no one is left unattended through their $24 / 7$ availability.

They have the potential to facilitate users immediate access to library services and collection at their convenience, fufilling the 5 Laws of Library Science as propounded by Padam Shree, Dr.S. R. Ranganathan, the Father of Library Science in India.

\section{Conclusion:}

Although chatbots often underwhelmed users initially, they will improve rapidly and offer a variety of benefits to business, including capacity for personalization, high engagement via push notifications, productivity via automation, revenue generation and access to consumers on wildly popular apps. Although bot technology has evolved significantly over the years, it has maintained core characteristics like an automated backend that retrieves and delivers information in response to inputs from an end user. Libraries can adopt conversational AI platforms to boost their existing relationship with their patrons in the new normal era. It has been observed that many users prefer messaging over other forms of communication. Thus making a case for the adoption and implementation of conversational chatbots empowered with deep learning, NLP and AI. They offer an easier, less intrusive and quicker than using other communication channels. Conversational AI enables libraries able to hold seamless, synchronous conversations with consumers across whatever channel they happen to be using at the time, no matter where they are. Importantly, those conversations could be a short one-off request or response, or part of a longer-running user engagement. Chat apps have attracted billions of users, and bots are the mechanism that enable organizations to deliver highly personalized interactions at scale. Improved personalization delivers a more relevant user experience, and the high levels of automation used by bots enable cost-effective delivery at scale. As a result, libraries can engage with patrons and 
achieve greater user satisfaction that typically outperform email and social networks. They attempt to comprehend the complexity of a human interaction, providing libraries a cost-effective way to handle and respond to the users in a responsible, authentic, accurate and meaningful way. They can complement and supplement the traditional as-well as virtual reference service offered by any library while additionally serving as a powerful marketing tool and a true and loyal patron companion.

\section{References:}

Cordny. (2020, February 13). Chatbot versus Virtual Assistantchatbot versus virtual assistant. Retrieved July 11, 2020, from https://testingsaas.nl/2020/02/13/chatbot-versus-virtualassistant/

Khurana, D., Koli, A., Khatter, K., \& Singh, S. (2017, August 17). Natural Language Processing: State of The Art, Current Trends and Challenges. Retrieved July 12, 2020, from https://arxiv.org/abs/1708.05148

King, D. L. (2018, October 2). Chatbots and Libraries. Retrieved July 14, 2020, from https:/davidleeking.com/chatbots-and-libraries/

Kore.ai. (2020a). Bring customers and brands together through personalized, omnichannel experiences. Retrieved July 12, 2020, from https://kore.ai/platform/features/omnichannel/

Kore.ai. (2020b). Channel Enablement. Retrieved from https://developer.kore.ai/docs/bots/channel-enablement/addingchannels-to-your-bot/

Kore.ai. (2020c). Enterprise Virtual Assistant Platform. Retrieved July 11, 2020, from https://kore.ai/

Kore.ai. (2020d). Publishing your Bot - Kore.ai Documentation. Retrieved July 13 , 2020 , from https//developer.kore.ai/docs/bots/publish/publishing-bot

Kore ai. (2020e). Universal Virtual Assistants; Orchestrate Tasks From Multiple Specialized Virtual Assistants. Retrieved July 12, 2020 . from kore.ai website: https:/kore,ai/platform/features/universal-bots' 
McNeal, M. L., \& Newyear, D. (2013, December 19). Chapter 1: Introducing Chatbots in Libraries. Library Technology Reports, 49(8), 5-10. Retrieved from https://joumals.ala.org/index.php/ltr/article/view/4504/5281

Smartsheet Inc. (2020). Artificial Intelligence Chatbots Are Changing the Way You Do Business and May Impact Your Bottom Line. Retrieved July 11, 2020, from https://www.smartsheet.com/artificial-intelligence-chatbots

Warschauer, M., \& Healey, D. (1998, April). Computers and language learning: an overview. Language Teaching, 31(2), 57-71. https://doi.org/10.1017/s0261444800012970

$>$ Yerram, R. (n.d.). How Small Talk Delivers a Great Deal by Elevating Chatbot Experience. Retrieved July 13, 2020, from https://blog.kore.ai/how-small-talk-delivers-a-great-deal-byelevating-chatbot-experience 